\title{
FLUXO DE OTIMIZAÇÃO EM PROJETO DE INOVAÇÃO UTILIZANDO FERRAMENTAS ESTATÍSTICAS EM CONJUNTO COM FORMULAÇÃO TEÓRICA
}

\author{
Cristiano Marques de Oliveira ${ }^{1}$ \\ ${ }^{1}$ Delphi Automotive Systems \\ E-mails: cristiano.m.oliveira@delphi.com
}

\section{RESUMO}

O desperdício relacionado à estratégia de tentativa \& erro nos projetos de inovação pode ter efeito negativo na competitividade, devido a atraso no lançamento e perda de qualidade. Nesse cenário, a aplicação das ferramentas estatísticas propostas pela metodologia seis-sigma, em conjunto com a formulação teórica, pode representar uma vantagem competitiva no desenvolvimento de soluções inovadoras. Esse trabalho tem como objetivo mostrar o fluxo de otimização utilizado no projeto de inovação relacionado à região do terminal elétrico desenvolvida para união com cabo através de solda por resistência, assim como os ganhos de conhecimento e qualidade obtidos durante o processo.

\section{INTRODUÇÃO}

Visando o aumento da competitividade, se torna necessário melhorar a confiança na realização de projetos, principalmente no desenvolvimento de novos produtos em projetos de inovação. Portanto, é necessário aumentar a competitividade de curto prazo no setor, sob o risco de uma presença cada vez maior de autopeças importadas sendo introduzidas nos veículos fabricados no Brasil [1].

Ainda como complemento da análise setorial, para manter e ampliar a posição competitiva nos próximos anos, as autopeças brasileiras deveriam focar o desenvolvimento em soluções voltadas ao mercado regional, com baixo custo e procurando integração e parceria estratégica com as montadoras [1]. Nesse cenário, surge a necessidade de uma estratégia mais robusta em comparação à tradicional baseada em tentativa \& erro que pode não ser suficiente para alcançar os resultados esperados de prazo e qualidade na introdução da inovação no mercado.

Tem-se como projeto de inovação, ou inovação tecnológica, a introdução no mercado de um produto (bem ou serviço) novo ou substancialmente aprimorado ou pela introdução na empresa de um processo novo ou substancialmente aprimorado [2].

A figura 1 [3] mostra a relação entre os tipos de projeto, incluindo o projeto de inovação, e o momento de oferta ao cliente ou introdução no mercado. 


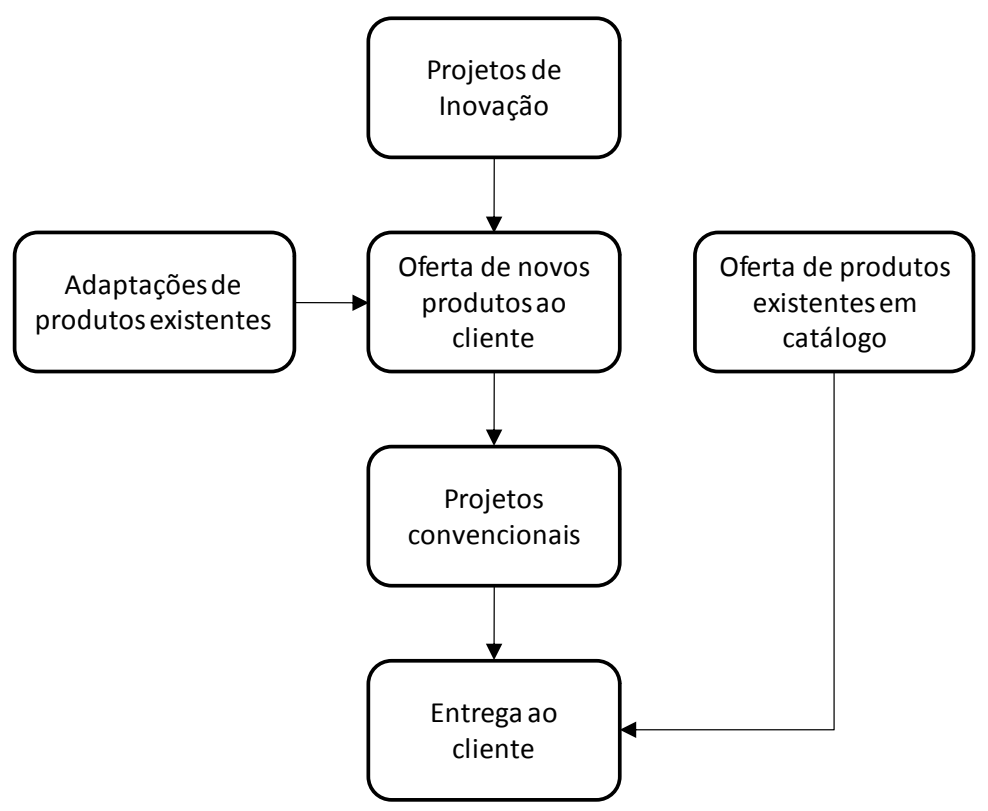

Figura 1: Tipos de projeto e oferta ao cliente [3]

Durante a execução desse tipo de projeto, a aplicação de formulações teóricas é muito útil para a busca do correto dimensionamento, porém em situação onde as diferenças nas condições das variáveis com relação ao modelo teórico são significativas, a sua aplicação pode requer um grau muito elevado de simplificação e adaptação que pode prejudicar a sua confiabilidade.

As ferramentas estatísticas de análise, propostas pela metodologia seis-sigma, podem auxiliar nos casos onde não existem formulações teóricas diretas para o problema estudado, mas o desconhecimento do modelo teórico pode levar a interpretações equivocadas dos resultados de experimentos. Portanto, o uso conjunto de ferramentas estatísticas e a formulação teórica pode representar um método poderoso para o desenvolvimento de soluções inovadoras.

\section{DFSS - SEIS-SIGMA EM PROJETOS DE INOVAÇÃO}

A metodologia seis-sigma propõe um modelo estruturado de execução do ciclo de melhoria e desenvolvimento, com o intuito de reduzir a variação através da utilização de ferramentas estatísticas para entender, analisar e controlar a relação entre as entradas e saídas do projeto.

O método seis-sigma tradicional é mais adequado para projetos melhoria incremental ou solução de problemas, para projetos de inovação o método DFSS, Design For Six-Sigma, é mais adequado devido ao foco no desenvolvimento de novos produtos e processos [4].

O DFSS é um método proativo que considera as melhores técnicas de prevenção de problemas nas fases iniciais do processo de desenvolvimento [5], com grande foco na voz do cliente, voz do negócio, entendimento da função do produto, modelamento e previsão do desempenho, desenho robusto, capacidade da manufatura e gerenciamento da variação. 


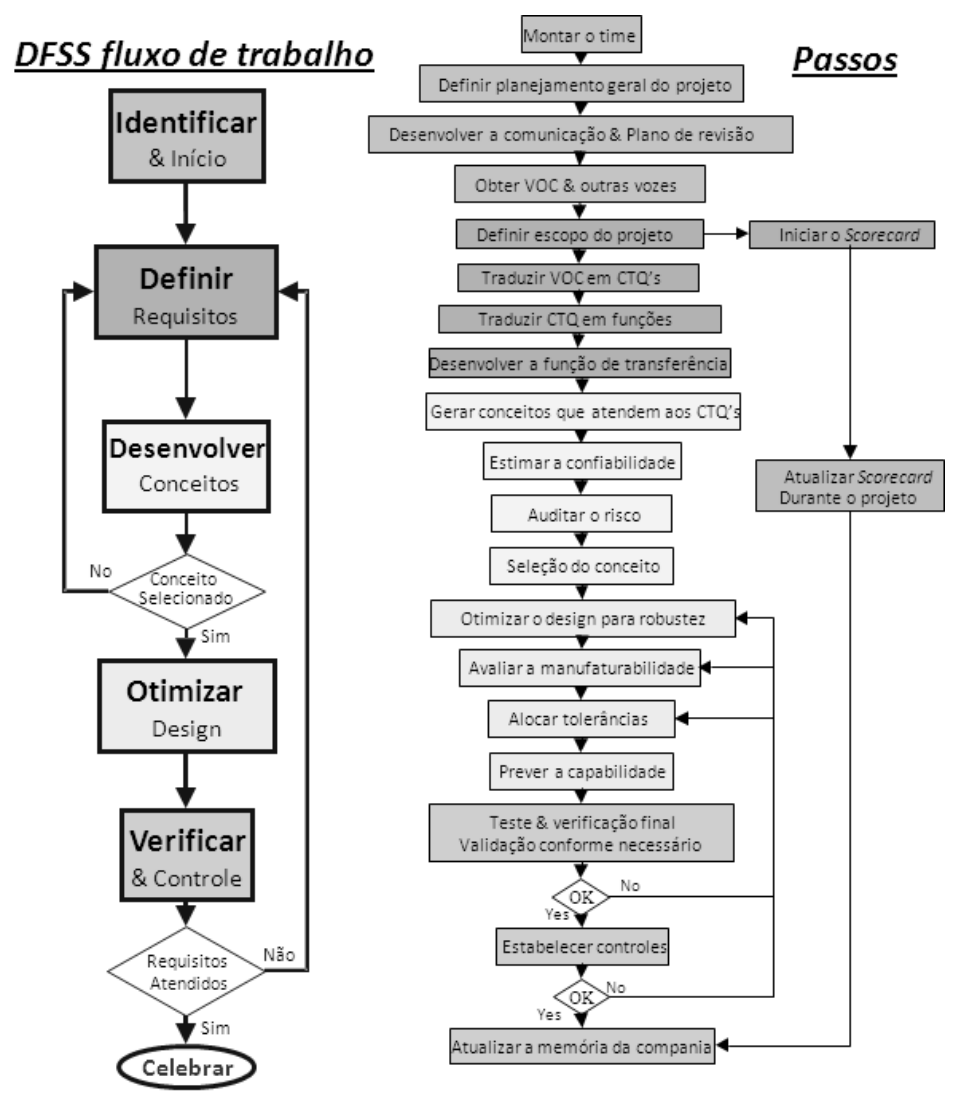

Figura 2: Etapas do Design For Six-Sigma [5]

A figura 2 ilustra uma estrutura de trabalho baseado no DFSS. As fases Identificar, Definir e Desenvolver fornecem as ferramentas necessárias para avaliação e seleção do melhor conceito, incluindo o mapeamento da voz do cliente e a definição dos elementos mensuráveis atrelados a ela. As fases Otimizar e Verificar têm como objetivo o detalhamento técnico do conceito selecionado, visando a otimização para a robustez e para atingir os objetivos iniciais do projeto [3].

\section{FLUXO DE OTIMIZAÇÃO}

Nesse trabalho é apresentado o fluxo de otimização utilizado durante o desenvolvimento da região de união entre cabo e terminal elétrico através de solda por resistência, para uso em chicotes elétricos automotivos.

Atualmente o método mais aplicado para essa união se baseia na conformação mecânica, onde o design da região de união dos terminais, chamada de garras de crimpagem, é amplamente conhecido e utilizado. Para a aplicação através de solda por resistência foi necessário o estudo de novas soluções com o intuito de garantir a busca pelo melhor design do terminal.

A solda por resistência é um processo termoelétrico cujo aquecimento é gerado na interface das peças a serem unidas pela da passagem de corrente elétrica através das peças por um tempo precisamente controlado e sob uma pressão ou força controlada. O nome solda por "resistência" deriva do fato que as resistências das peças e eletrodos são utilizadas para gerar o aquecimento nas suas interfaces [6]. 
A figura 3 exemplifica a diferença entre a região de união entre cabo e terminal elétrico de acordo com o método de aplicação, crimpagem por conformação mecânica e solda por resistência à passagem de corrente elétrica.
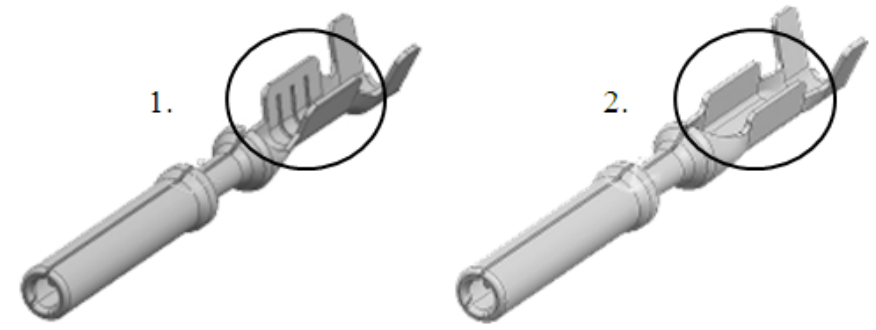

Figura 3: Região do terminal elétrico para união com cabo

1. Crimpagem mecânica; 2. Solda por resistência

Para o fluxo de otimização, além do formato e dimensões da região de união do terminal elétrico, também foram levados em consideração os parâmetros do processo de aplicação da solda por resistência, uma vez que esses parâmetros são determinantes nesse tipo de aplicação.

A variável de saída considerada para verificação da qualidade da aplicação foi resistência à tração da união soldada entre cabo e terminal elétrico. Essa variável foi selecionada pela facilidade de execução de testes e pela disponibilidade de especificações para referência de requisito mínimo a ser atingido, atualmente usado pelas montadoras para uniões por crimpagem.

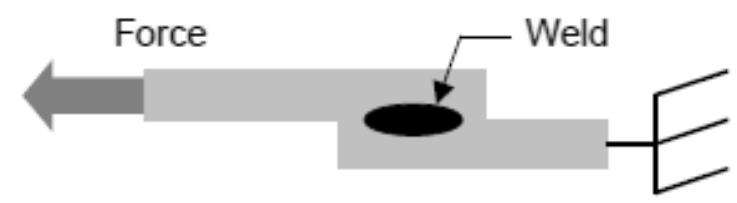

Figura 4: Teste de resistência mecânica da solda [7]

\subsection{APLICAÇÃO DAS FERRAMENTAS ESTATÍSTICAS}

O fluxo de otimização foi iniciado com a aplicação de testes de hipótese com o intuito de verificar o impacto do formato da seção transversal da região de união e do comprimento da solda na sua resistência à tração.

A ferramenta estatística teste de hipótese tem o propósito de estabelecer uma base para que se possam obter evidências que reprovem a declaração ou a aceitem como verdadeira. Uma hipótese é uma suposição feita, com o objetivo de se testar suas consequências lógicas ou empíricas [8].

$\mathrm{Na}$ análise dos formatos, foi observado que o modelo proposto com base plana e paredes paralelas apresentou resistência mecânica estatisticamente maior que o formato com base arredondada e paredes inclinadas. 


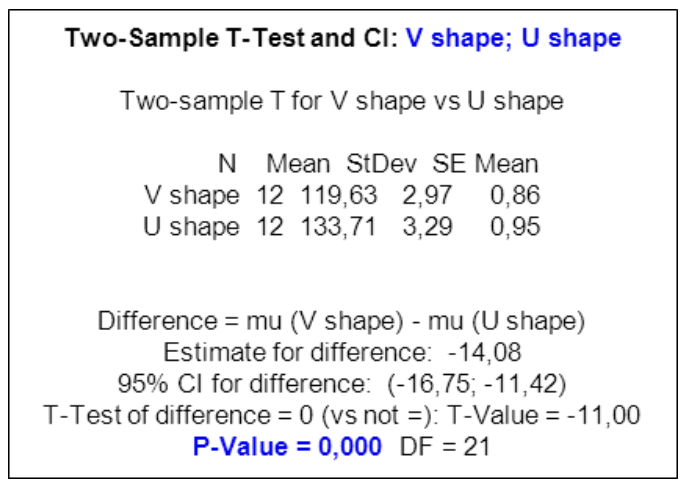

Figura 5: Teste de hipótese do formato

Os comprimentos considerados para o testes de hipótese foram determinados de acordo com os limites envolvidos para os processos de aplicação da solda e da fabricação ou adaptação dos terminais elétricos. Essa análise estatística não identificou diferença significativa entre os limites de comprimento, logo a menor dimensão pode ser adotada por requerer menor energia para solda.

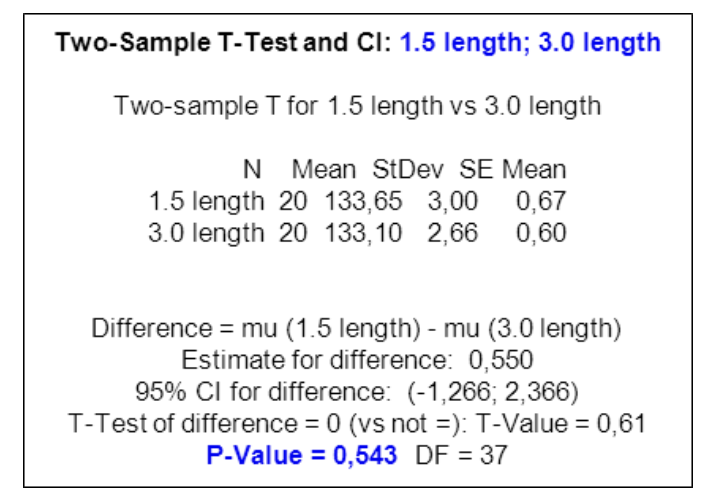

Figura 6: Teste de hipótese do comprimento

Após a análise dos parâmetros geométricos do terminal, foi iniciado o estudo levando em consideração as variáveis do processo de aplicação.

Além da corrente elétrica aplicada para promover o aquecimento, outros fatores fundamentais para o processo de solda por resistência são o tempo de solda, a força de compressão, o deslocamento do eletrodo, materiais envolvidos, entre outros [6].

Para essa etapa da análise, foi realizado um estudo de regressão onde a variável de entrada selecionada foi a corrente elétrica aplicada, por ser ajustável e poder representar a energia envolvida no processo de forma significativa.

No estudo de regressão investiga-se a dependência da característica Y, variável dependente ou regressão, em uma ou mais características $X$, variável independente ou regressora. $\mathrm{O}$ cálculo da correlação serve para expressar em um coeficiente de medida estatística a força da relação entre duas variáveis em questão [8].

No estudo apresentado nesse trabalho foi observado que o processo apresenta correlação forte e uma grande região robusta de aplicação a partir de uma determinada corrente ou energia, ou seja, mesmo com grande variação na entrada, característica $X$, pouca variação foi observada na variável de saída, característica Y, em uma determinada região. 


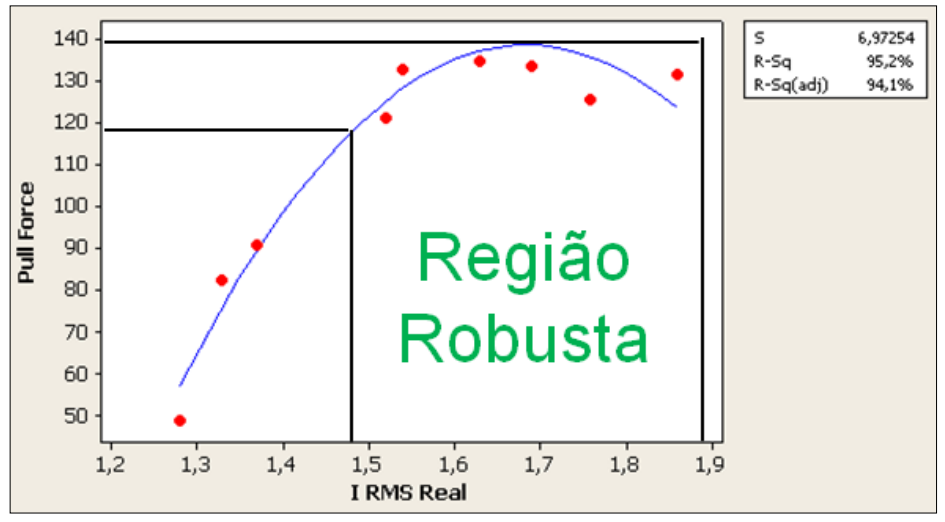

Figura 7: Estudo de regressão e região robusta

\subsection{RELACIONAMENTO COM A FORMULAÇÃO TEÓRICA}

Do modelo obtido através da regressão foi possível encontrar a faixa de corrente para atingir a região robusta de resistência à tração de uma aplicação realizada através da solda por resistência. Para obter a relação com a quantidade de energia mínima para uma aplicação robusta, foi utilizada a equação geral da geração de calor para solda por resistência [6].

$$
Q=I^{2} \times R \times t \times K
$$

Onde, "I" é a corrente para solda que atravessa as peças, "R" é a resistência elétrica das peças, " $t$ " é o tempo de solda e "K" é o fator térmico. A corrente de solda e a duração são controladas pela fonte de potência da solda por resistência. A resistência das peças é função da força de solda e dos materiais utilizados. O fator térmico pode ser afetado pela geometria das peças, fixações e força de solda [6].

Como se pode notar, as variáveis corrente, resistência e tempo apresentadas na equação geral da geração de calor (1) podem ser monitoradas e/ou medidas em cada aplicação, porém o fator térmico " $K$ " não é definido ou tabelado sendo dependente de cada configuração.

Para esse estudo o fator " $K$ " foi definido em função da massa das peças a serem soldadas e os parâmetros que podem ser medidos no processo, através do relacionamento entre os dados do estudo estatístico de regressão com as equações da geração de calor (1) e da transferência de energia (2) da formulação teórica.

$$
Q=m \times c \times \Delta T
$$

Onde, "m" é a massa das peças a serem soldadas, "c" é o calor específico dos materiais, para esse trabalho foi considerado o cobre e suas ligas, e " $\Delta \mathrm{T}$ " é a elevação de temperatura até a temperatura de fusão [9].

Com as informações das dimensões do terminal e do eletrodo, junto com a bitola do cabo, é possível determinar a massa a ser soldada em cada configuração de aplicação. Dessa 
forma, ambos os dados estatísticos, obtidos do estudo de regressão, e teóricos estavam definidos para que o relacionamento pudesse ser estabelecido.

\section{RESULTADOS}

Após o relacionamento dos dados estatístico com as equações da formulação teórica, foi possível desenvolver um método de cálculo para estimar a energia mínima necessária para atingir o patamar robusto de resistência à tração. Com o valor de energia calculado, foi possível estimar o parâmetro de corrente elétrica para a aplicação de diferentes configurações de terminal e cabo, mesmo que essa não tenha sido testada anteriormente, mantendo os demais parâmetros do processo.

As aplicações com os parâmetros de corrente estimados foram testados para diversas configurações, variando a bitola dos cabos e diferentes tipos terminais com espessuras, materiais e dimensões variadas. Para as aplicações com cabos de cobre puro, todos os resultados de resistência à tração foram altamente robustos, mostrando altos níveis de capabilidade de processo nos estudos realizados.

Também foi realizado teste de resistência à tração na solda com cabo elétrico de bitola reduzida fabricado em liga de cobre endurecida, aplicação realizada com a corrente estimada por cálculo e mesmo tempo de solda das aplicações com cabo de cobre puro. Para essa configuração, os resultados obtidos de resistência à tração ficaram abaixo do obtido com cabos de cobre puro, mostraram assim oportunidade para melhoria.

\section{EXPERIMENTOS ADICIONAIS}

A estratégia utilizada para o estudo adicional dos parâmetros de aplicação com cabos de bitola reduzida fabricado em liga de cobre endurecida foi baseada na ferramenta estatística DOE, Design Of Experiments, que consiste em selecionar fatores e níveis que serão combinados em diversas condições experimentais para quantificar o efeito dos fatores e interações na saída medida [5]. Essa ferramenta tem como objetivo maximizar o conhecimento obtido através de dados experimentais [10].

Para poder testar as combinações de corrente e tempo mantendo o nível de energia transferida constante, foi utilizado o tipo de experimento DOE de Mistura, onde os níveis dos fatores são definidos de forma proporcional. Logo foi possível definir níveis para os fatores de corrente e tempo de forma consistente com a formulação teórica para que a energia transferida fosse constante.

O nível constante de energia foi definido através do cálculo mostrado anteriormente, desenvolvido através do uso conjunto das ferramentas estatísticas e formulação teórica. Essa quantidade de energia leva em consideração o patamar robusto de resistência à tração, a massa a ser soldada e o aumento de temperatura. Para mantê-la constante variando a corrente e o tempo, foi necessário desenvolver uma equação de transformação (3), pois a corrente varia de forma quadrática enquanto o tempo é um multiplicador simples.

$$
I_{\text {doe }}=\sqrt{\frac{Q_{\text {cte }}}{R^{\prime} \times k^{\prime} \times t_{\text {doe }(-,+)}}} \rightarrow I \%+t \%=100 \%
$$


Duas hipóteses foram formuladas para análise, a diminuição do tempo com o aumento proporcional da corrente e o aumento do tempo com a diminuição proporcional da corrente. $\mathrm{O}$ aumento e a diminuição dos fatores foram definidos em relação à configuração inicial, onde foi aplicada a corrente estimada por cálculo e o mesmo tempo de solda usado para aplicações com cabos de cobre puro.

Foram realizados experimentos consecutivos com diferentes configurações dos fatores para a busca da configuração de máxima resistência à tração, dessa forma foi possível encontrar os níveis de tempo e corrente com resultado significativamente melhor que a condição inicial.

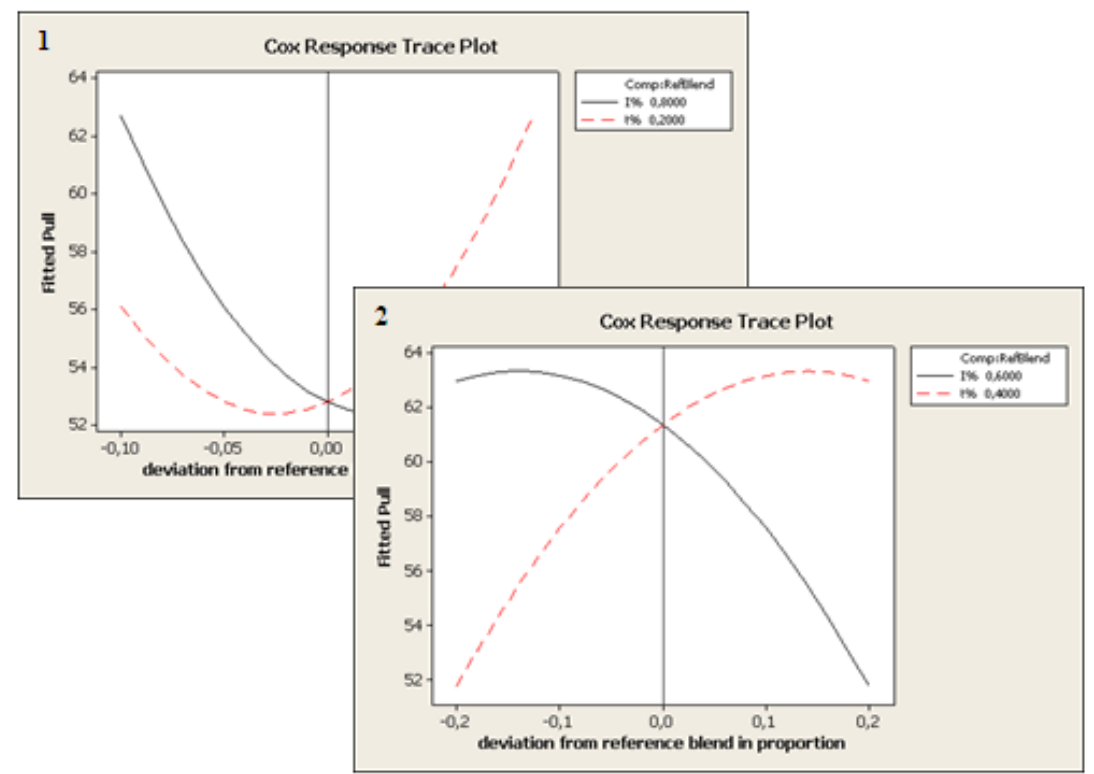

Figura 8: Experimentos consecutivos (DOE de Mistura)

1. Diminuição do tempo com aumento da corrente;

2. Aumento do tempo com diminuição da corrente

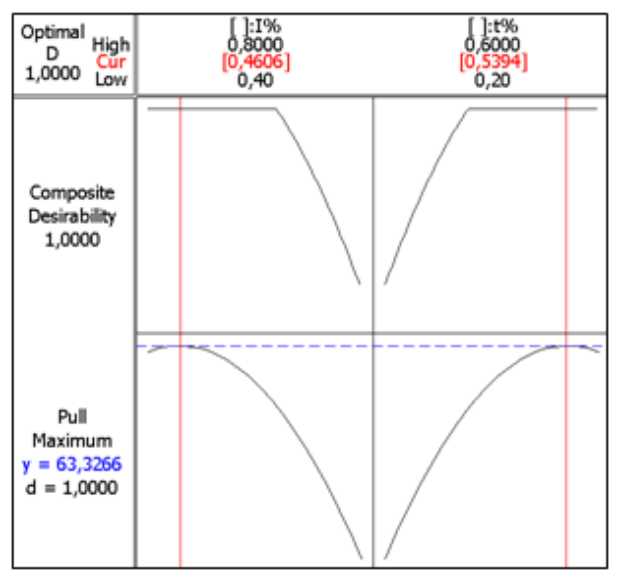

Figura 9: Otimização dos parâmetros

Com o modelo estatístico obtido desse experimento foi possível encontrar os parâmetros otimizados de corrente e tempo de solda, assim como confirmar o desempenho dessa configuração através do estudo de capabilidade. 


\section{CONCLUSÃO}

O uso de ferramentas estatísticas em conjunto com a formulação teórica foi fundamental para o sucesso do fluxo de otimização apresentado, uma vez que os resultados obtidos de cada estratégia foram utilizados para alimentar a outra de forma sucessiva.

Ambas representam simplificações com relação ao modelo real, dessa forma a abordagem seis-sigma teve peso significativo para garantir e aumentar a confiança nos resultados obtidos.

Como complemento às estratégias apresentadas, existe a oportunidade da aplicação da análise de elementos finitos. Essa poderia representar um ganho importante, porém com atenção ao esforço e recursos necessários para múltiplas simulações das diversas configurações, semelhante aos experimentos reais e cálculo com modelo teórico.

Para esclarecimentos adicionais, contatar:

Cristiano Marques de Oliveira

Delphi Automotive Systems

Phone: +55 (012) 3978-2006

E-Mail: cristiano.m.oliveira@ delphi.com

\section{REFERÊNCIAS}

[1] ABDI, "Estudo Prospectivo Setorial Automotivo, Brasília," 2009.

[2] GRIZENDI, Eduardo, "Manual de Orientações Gerais Sobre Inovação,” MRE, 2011.

[3] OLIVEIRA, Cristiano M., "Lições aprendidas no planejamento dos projetos de inovação na área de Engenharia Avançada," SAE Technical Paper 2011-36-0230, 2011.

[4] YANG, Kai and El-Haik, Basem, "Design for Six Sigma A Roadmap for Product Development," McGraw-Hill, 2003.

[5] DELPHI, "DFSS Black Belt - Material de Treinamento," Setembro 2009.

[6] MIYACHI, "Fundamentals of Small Parts Resistance Welding," 2006.

[7] STEINMEIER, David, "Weld Joint Testing Basics," microJoining Solutions, microTips.2004.10.20.

[8] DELPHI, "Seis-Sigma Black Belt - Material de Treinamento," Setembro 2007.

[9] CENGEL, Yunus, "Heat Transfer: A Practical Approach," Mcgraw-Hill, 2nd edition, 2002.

[10] SCHMIDT, Steve PhD, "Communicating Design of Experiments (DOE) to Nonstatisticians," Air-Academy, 2002.

\section{DEFINIÇÕES/ABREVIAÇÕES}

DFSS: Projeto para Seis Sigma (Design for Six Sigma). Método proativo que considera as melhores técnicas de prevenção de problemas nas fases iniciais do processo de desenvolvimento [5].

DOE: Projeto de Experimentos (Design of Experiments). Método para maximizar o conhecimento obtido através de dados experimentais [10]. 